\title{
Performance Analysis of Image Processing in Hexagonal Grid Based on Image Compression, Denoising and Steganography
}

\author{
Jeevan K. M., Padmaja V. K., Anne Gowda A. B., S. Krishnakumar
}

\begin{abstract}
This paper gives a summary of different works we had performed in hexagonal grid for the performance analysis of image processing in hexagonal grid. Processing based on hexagonal grid is a new approach in image processing because of its various advantages. This paper covers image compression and image denoising in hexagonal grid. Image compression using wavelet transform is performed in hexagonal grid. The performance of compression in hexagonal grid and conventional rectangular grid are analyzed in terms of Mean Square Error (MSE) and Peak Signal to Noise Ratio (PSNR). It is observed that better PSNR is obtained in the case of hexagonal grid. Wavelet thresholding based method is done for image denoising in hexagonal grid. The thresholding method is named as 'Jeevkrish' method, which is the modification of NeighShrinkthresholding method. The performance is analyzed using MSE, PSNR and Structural Similarity (SSIM) Index. An algorithm ('JK' algorithm) for generating a pseudo hexagonal structure image and a method for image hiding using the pseudo hexagonal image as the cover image are also discussed in this paper
\end{abstract}

Keywords: Hexagonal Image, Gabor Filter, Compression, Denoising, Steganography

\section{INTRODUCTION}

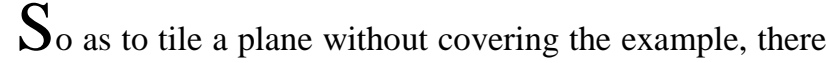
exist three decoration plans [1]. They are the decoration with squares, regular triangles and hexagons. Decoration with hexagon is the most able decoration group among them in view of its various advantages like smaller quantization error, equidistance, consistent connectivity, higher symmetry and greater angular resolution. The human retina (some portion of human eye) intently takes after a hexagonal framework space. The poles and cones cells in fovea (some portion of the retina) are arranged in a hexagonal manner than a rectangular one. This is another inspiration for utilizing hexagonal imageprocessing. In the animal vision additionally the plan of rods and cones are having hexagonal decoration. So by demonstrating and preparing images on a hexagonal framework space, one can get the common conduct to

Revised Manuscript Received on December 15, 2019

* Correspondence Author

Jeevan K. M.*, Dept. of Electrical, Electronics and Communication Engineering, GITAM Deemed to be University, Bangalore Campus, Karnataka, India.

E-Mail: jeevanjeevan77@gmail.com, jeevan.mani@gitam.edu.

Padmaja V. K., GITAM School of Technology, Banglore, Karnataka, India.

Anne Gowda A. B., GITAM School of Technology, Banglore, Karnataka, India.

S. Krishnakumar, Mahatma Gandhi University, Kottayam, Kerala, India. E-Mail: jeevanjeevan77@gmail.com acknowledge computer vision.

Objective of our work is to analyze, whether the hexagonal representation improves the quality of images and image processing algorithms. This work is concentrated on image compression and denoising in hexagonal grid. Also, an algorithm is proposed for generating a pseudo hexagonal structure image and an image hiding algorithm using this pseudo hexagonal structure image as cover image.

The problems addressed in our work are summarized as follows:

- Two main areas concentrated in this work are image compression and image denoising. Performance analysis of image compression and denoising in hexagonal grid compared with the performance of compression and denoising in conventional square grid image.

- The hardware is not available for capturing Hexagonal images. This is one of the difficulties faced by the researchers in this area. Therefore we have to convert rectangular to hexagonal image in order to perform hexagonal image processing. Therefore, simulation of pseudo hexagonal pixel image is addressed in this paper.

- An image hiding technique, as an application of pseudo hexagonal pixel image, is also included in this work.

\section{PERFORMANCE ANALYSIS OF HEXAGONAL IMAGE PROCESSING}

Image compression and denoising are the two areas concentrated in this work for the performance analysis of hexagonal image processing. In addition to compression and denoising, simulation of pseudo hexagonal pixel image and an image hiding technique, as an application of pseudo hexagonal pixel image, are also included in this work. As there is no hardware available for acquiring hexagonal images, conversion, from conventional image, has to be performed for processing images in hexagonal domain Throughout this work alternate pixel suppressal method is used for simulating hexagonal sampled grid. We also used Gabor filer for image enhancement and interpolation.

Using alternate pixel suppressal method, proposed by PramodSankar et.al [3], hexagonal grid can be simulated on rectangular grid. The sub-sampling procedure is given by the equation 1 


$$
\begin{aligned}
& \text { pixel_val } \text { hex }(i, j) \\
& =\left\{\begin{array}{c}
\text { pixel_val }(2 * i, 2 * j), \text { if iiseven } \\
\text { pixe_val }(2 * i, 2 * j+1), \text { ifiisodd }
\end{array}\right.
\end{aligned}
$$

According to this method; "The entire pixels in the conventional grid which do not associated with their hexagonal matching part are suppressed. Suppression of the pixel values indicate that the pixel values are assigned to zero. When processing this sub-sampled image, we do not consider the suppressed pixels for computation". The hex image simulated using this method has only one-fourth of the number of pixels in the rectangular grid. Because of this reason the results of image processing in hexagonal domain is not comparable to that over the regular rectangular lattice on which, we simulated the Hexagonal Pixel Grid (HPG). Therefore we have to consider a rectangular lattice that has the same resolution as that of the modeled hex lattice.
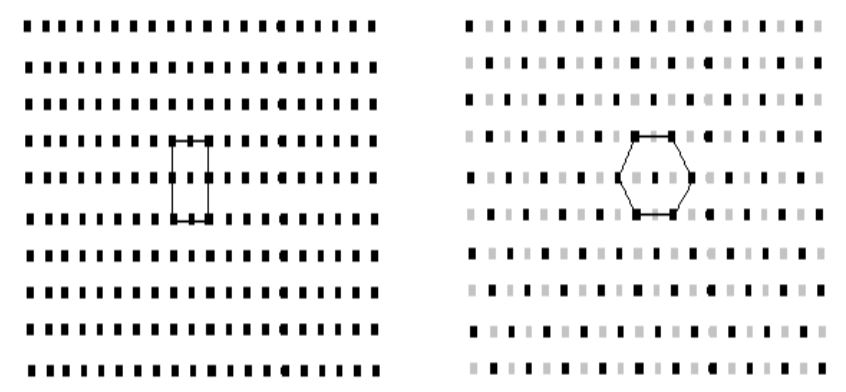

Fig.1 (a) Rectangular grid (b) Simulated Hexagonal grid [9]

\section{A. Compression in Hexagonal Domain using Wavelet}

The block diagram for wavelet based image compression in hexagonal grid [4] is shown in Fig.2.

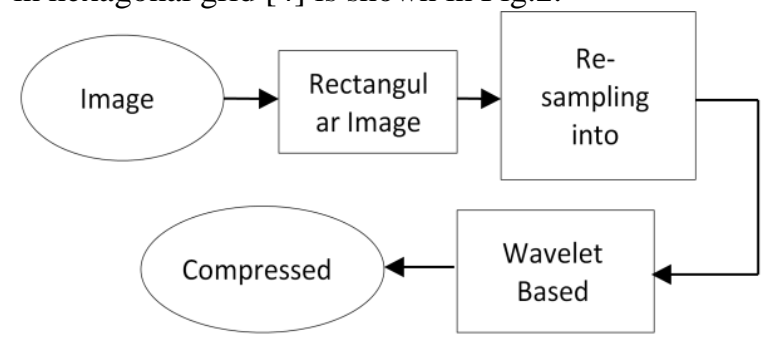

\section{Fig.2 Block Diagram for Wavelet Based Compression on Hexagonal Sampling Grid}

The steps used for compression [10] can be summarized as:

- Using alternate pixel suppressal method the input image is re-sampled to get image in hexagonal lattice.

- Performed interpolation using Gabor filter to improve the quality of image represented in hexagonal lattice. The interpolation steps are as follows

- Hexagonal structure exhibits directional symmetry in $0^{\circ}, 60^{\circ}$ and $120^{\circ}$ orientations.

- Select orientation of Gabor filter along $0^{0}$, $60^{\circ}$ and $120^{\circ}$, and perform filtering in above mentioned orientations.

- The filtered image is superimposed to get the interpolated image.

- The interpolated image is then decomposed using wavelet.

- Perform the compression using the MATLAB function 'wdencmp'

\section{B. Denoising in Hexagonal Domain}

An image denoising method using modified NeighShrinkthresholding method, named as JeevKrish[5] (Jeev: first four letters of the name of the first author, Krish: first five letters of the name of the fourth author) thresholding is explained in this section. The Fig. 3 shows the block diagram for the denoising method.

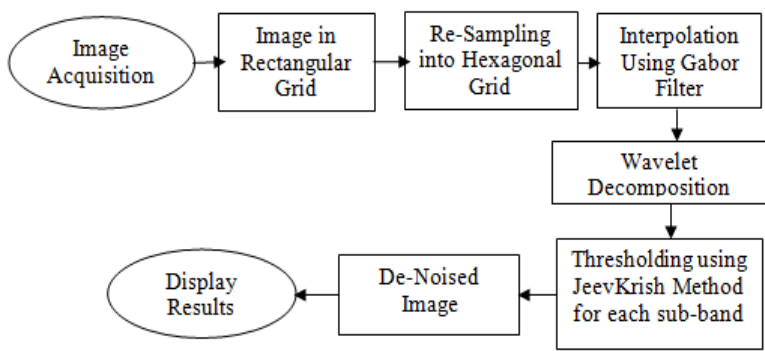

Fig. 3 Block Diagram for the Denoising Method

As there is no hardware to acquire an image in hexagonal domain, we have to re-sample the image in rectangular domain to hexagonal domain for further processing. The hexagonal domain image is then interpolated using Gabor filter.

The filtered image is then decomposed using wavelet to get four sub-bands viz. LH, HL and HH, where LL is the low frequency sub-band, and most of the image details are present in this sub-band. LH, HL and HH contain high frequency components and hence, most of the noises are present in these bands. Therefore, thresholding is done on all the coefficients in $\mathrm{LH}, \mathrm{HL}$ and $\mathrm{HH}$ sub-bands. For example, let $\mathrm{C}_{\mathrm{ij}}$ be the wavelet coefficients in the sub-band to be threshold. Select the neighborhood window with size $3 \mathrm{X} 3$ with $\mathrm{C}_{\mathrm{ij}}$ as the center element. Then calculate the parameter represented as $S_{i, j}^{2}$, which is the sum of the squares of coefficients present in the selected window. Then the thresholding is done using JeevKrishthresholding method proposed in [11].

\section{Simulation of Pseudo Hexagonal Image}

Image with pseudo hexagonal pixels was proposed by Wuthrich and Stucki [6]. This helps us to compare the visual effect of square and hexagonal pixels.

Yabushita and Ogawa [7] implemented pseudo hexagonal element which consist of square pixels with an aspect ratio of $12: 14$. 
This Section describes pseudo hexagonal structure simulation with a different ratio of $9: 8$. The name of the algorithm is given as ' $\mathrm{JK}$ algorithm' [8]. We used the first letters of the first author (J) and third author (K) for naming the algorithm.

In this algorithm the pseudo hexagonal pixel is produced from the square pixels. Each square pixel is changed over to relating hexagonal pixel. So as to build the hexagonal pixel from the square pixel, the square pixel is represented as a $9 \mathrm{X}$ 8 matrix as appeared in the Fig.4. That is, from the single pixel, 72 pixels are produced and the gray scalevalue of the whole 72 pixel is taken as that of the square pixel chose. The 72 pixels are then named as sub pixels. As delineated in the Fig.4, we are utilizing the sub pixels named as '1' for making a hexagonal pixel and different pixels, which are set apart as 0 are disposed of.

In the same manner the each pixels in the given image can be converted to pseudo hexagonal pixels. Each hexagonal pixel obtained is then combined in such a manner to get the image with pseudo hexagonal pixels.



Fig.4 Pseudo Hexagonal Pixel Using Square Pixel

\section{Image Hiding Using Pseudo Hexagonal Image}

In the proposed image concealing strategy, the pseudo hexagonal cover image, which is produced utilizing JK calculation, is utilized. Utilizing the proposed technique, the whole bits of the secret image can be inserted in the cover image. Dissimilar to the normal LSB substitution strategy for concealing method, in proposed technique every single bit in the pixels are implanted in the cover image without disposing of a solitary bit. The accompanying area discloses how to hide one pixel of secret image to the pseudo hexagonal cover image. Let $\mathrm{C}(\mathrm{I}, \mathrm{j})$ be one pseudo hexagonal pixel with gray level 220, which is appeared in Fig.5

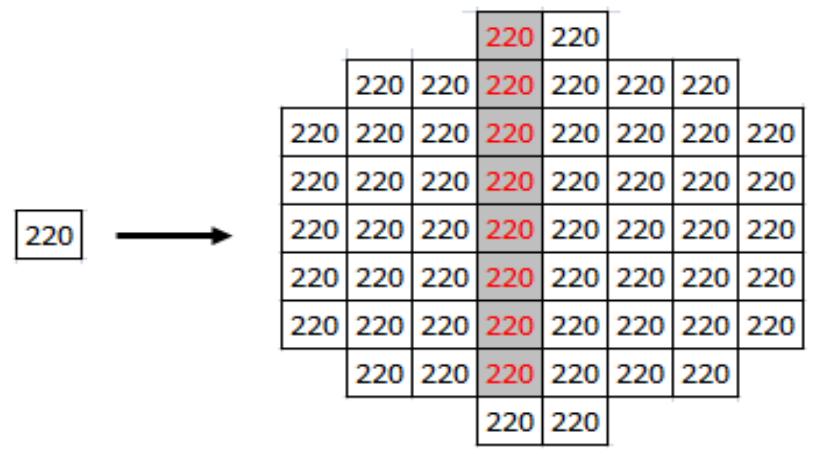

Fig.5 Pseudo hexagonal pixel with gray level value 220
Let $S(i, j)$ be one pixel of the secret image with gray level value 98 . The binary representation of $C(i, j)$ and $S(i, j)$ is shown in Fig.(6). The each bit of the selected pixel is then replaced with the LSB of any of the eight sub pixels of pseudo hexagonal pixel of the cover image. In this example LSB of the highlighted sub pixels (Fig. 5) are used [9].

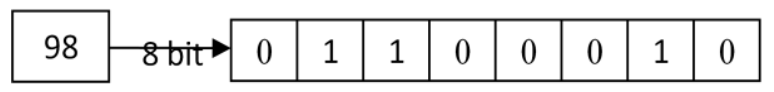

(a)

\begin{tabular}{|c|c|c|c|c|c|c|c|c|c|}
\hline 22 & 8 bit & 1 & 1 & 0 & 1 & 1 & 1 & 0 & 0 \\
\hline
\end{tabular}

(b)

Fig.6 Binary representation of (a) 98(one pixel of secret image) (b) 220 (one pixel ofcover image).

\section{RESULTS AND DISCUSSION}

This section presents a detailed description of results obtained and analysis of each work mentioned in section 2 .

\section{A. Results and Discussion on Wavelet Based Compression}

The compression is performed on hexagonal grid image as well as the conventional image. MSE and PSNR are used for the performance comparison. The experimental results show that the hexagonal representation gives better results in terms of MSE and PSNR. The overlapping nature of wavelet transform reduces the blocking artefacts, which is the drawback of DCT based compression. WT based compression gives improvement in quality of the image at higher compression ratio (CR). This is due to the superior energy compaction properties of WT [16, 17]. Hexagonal representation also gives compression, as it required less number of pixels to represent the same amount of data than the conventional representation.

Table 1 gives the performance analysis of wavelet based compression in terms of MSE and PSNR for hexagonal as well as rectangular grid images.

\begin{tabular}{|c|c|c|c|}
\hline Test Images & Sampling & MSE & PSNR \\
\hline \multirow{2}{*}{ Barbara } & Hexagonal & $\mathbf{1 3 . 3 7}$ & $\mathbf{3 8 . 8 8}$ \\
\cline { 2 - 4 } & Rectangular & 23.78 & 37.69 \\
\hline \multirow{2}{*}{ Lena } & Hexagonal & $\mathbf{3 . 8 5}$ & $\mathbf{4 2 . 2 8}$ \\
\cline { 2 - 4 } & Rectangular & 12.50 & 37.16 \\
\hline Fishingboat & Hexagonal & $\mathbf{1 2 . 8 0}$ & $\mathbf{3 7 . 0 6}$ \\
\cline { 2 - 4 } & Rectangular & 22.51 & 34.61 \\
\hline
\end{tabular}

\section{B. Results and Discussion on Denoising}

The proposed JeevKrishdenoising method is performed on many images. The same is performed on the conventional rectangular lattice images for comparison. The performances of proposed method on conventional images are better than the denoising using NeighShrink, VisuShrink and SureShrink methods. 
It also performed better than the MATAB de-noising function 'wdencmp'.

The interesting part of the work is that if we represent the images in hexagonal lattice, the performance of the proposed method again improves and gives better Mean Square Error (MSE) and Peak Signal to Noise Ratio (PSNR). These results confirm that the hexagonal representation is better than the conventional rectangular lattice.

In NeighShrink, $S_{i, j}^{2}$ obtained is compared with $\mathrm{T}_{\mathrm{u}}^{2}$, where $\mathrm{Tu}$ is the universal threshold which does not deals with minimizing MSE and hence eliminate many coefficients. But in the case of JeevKrishthresholding, the proposed method $S_{i, j}^{2}$ is compared with $\mathrm{T}_{\text {sure }}^{2}$, the SureShrink threshold. It is based on Stein's Unbiased Risk Estimator (SURE) and it minimizes mean squared error and gives an optimal threshold value which is better than Tu. In JeevKrish method, each coefficient in each sub-band is thresholded using this optimal threshold value. We are also considering the effect of neighbor coefficients for thresholding the coefficient $\mathrm{Ci}, \mathrm{j}$. So this method gives better results than other methods. The obtained PSNR for five different images for different noise level are represented in Table 2.

Table 2: PSNR for different noise level

\begin{tabular}{|c|c|c|c|c|}
\hline \multirow{4}{*}{$\begin{array}{c}\text { Noise } \\
\text { Level } \\
(\boldsymbol{\sigma})\end{array}$} & $\begin{array}{c}\text { Test } \\
\text { Images }\end{array}$ & $\begin{array}{c}\text { MATLAB } \\
\text { Fnction } \\
\text { (wdencmp) }\end{array}$ & \multicolumn{2}{|c|}{$\begin{array}{c}\text { JeevKrish (Proposed } \\
\text { Method) }\end{array}$} \\
\cline { 3 - 5 } & & $\begin{array}{c}\text { Rectangular } \\
\text { Grid Image }\end{array}$ & $\begin{array}{c}\text { Rectangular } \\
\text { Grid Image }\end{array}$ & $\begin{array}{c}\text { Hexagonal } \\
\text { Grid } \\
\text { Image }\end{array}$ \\
\hline \multirow{4}{*}{10} & car.jpg & 35.68 & 36.46 & $\mathbf{4 3 . 8 7}$ \\
\cline { 2 - 5 } & elephant.jpg & 33.33 & 35.55 & $\mathbf{3 8 . 2 8}$ \\
\cline { 2 - 5 } & barbara.png & 32.01 & 34.02 & $\mathbf{3 6 . 9 7}$ \\
\cline { 2 - 5 } & lena.png & 34.41 & 35.13 & $\mathbf{4 2 . 3 7}$ \\
\hline \multirow{4}{*}{20} & car.jpg & 31.78 & 31.85 & $\mathbf{4 3 . 1 4}$ \\
\cline { 2 - 5 } & elephant.jpg & 31.53 & 31.88 & $\mathbf{3 7 . 4 3}$ \\
\cline { 2 - 5 } & barbara.png & 30.51 & 30.9 & $\mathbf{3 6 . 5 5}$ \\
\cline { 2 - 5 } & Lena.png & 31.44 & 31.45 & $\mathbf{4 1 . 4 2}$ \\
\hline
\end{tabular}

In order to construct a hexagonal pixel, JK algorithm uses 56 sub pixels of square shape. Hence it is considered as pseudo hexagonal pixels. The image created using these pseudo hexagonal pixels satisfying consistent connectivity and six neighbours properties of hexagonal structure.

The Fig. 8 represents the central pixel and its 6 neighbours in pseudo hexagonal image. From the figure it can be identified that the central pixel which is represented as ' 0 ' have six neighbours represented as 1 to 5 . The distance to the each pixel can be calculated as follows.

Consider the neighbour pixel ' 3 '. The distance from the centre of the central pixel to the centre of pixel 3 and 6 is 8 units (Fig.8). The distance to the center of the other neighbor pixels (pixels 1, 2, 4 \& 5) from the centre of center pixel can be calculated as shown below.

Consider the pixel 2 is explained here. The line drawn

\section{Discussion on JK Algorithm}

between the centers of pixel ' 0 ' and ' 2 ' can be considered as the hypotenuse of the right angled triangle as shown in the Fig.8. The length of this line determines the distance between these pixels.

The distance can be calculated using the trigonometric identity. Therefore, the distance from the central pixel to pixel number 2 is

$$
\sqrt{7^{2}+4^{2}}=8.06 \cong 8 \text { units }
$$

Where, 4 and 7 represents the distance measured from the central pixel to the starting of third pixel from the starting of the $3^{\text {rd }}$ pixel to the center of $2^{\text {nd }}$ pixel respectively.

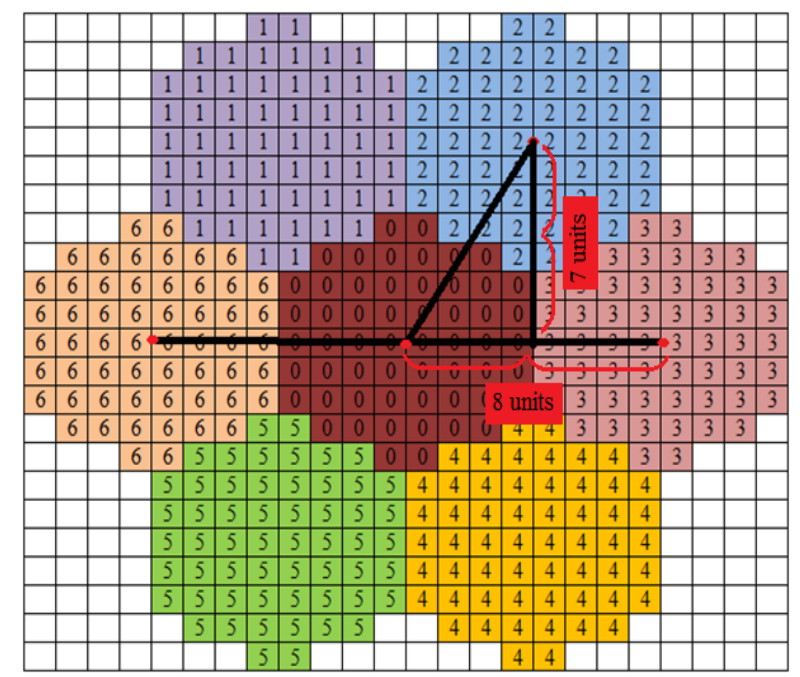

\section{Fig.8 Six Neighbors of Hexagonal Pixel}

\section{Results and Discussion on Image Hiding}

The process of hiding or embedding the secret image into the cover image is named as image Steganography. In the current techniques, the whole bits of secret image are not inserting in the cover image. This prompts quality misfortune in the secret image while recouping from the stego image. One answer for this issue is to implant whole bits of the secret image. On the off chance that it is conceivable, we can monitor the nature of the secret image which is covered up or implanted in the cover image.

In this proposed strategy, every single bits of the secret image are installing in the cover image and it is conceivable to extricate all the implanted bits with no distortion. Along these lines, the nature of the image is preserved $100 \%$. For the exhibition examination of the proposed strategy, we utilized MSE and SSIM. We determined the MSE between the secret image before inserting and the secret image taken out from the stego image. The MSE acquired is zero. We likewise determined the SSIM between the secret image before installing and the image recovered from the stego image. The SSIM acquired is solidarity. This implied the nature of the image is preserved $100 \%$.

We likewise estimated the SSIM index and MSE between the pseudo hexagonal coverimagebefore and after implanting the secret image. MSE is seen as less and SSIM is unity. These outcomes shows that the algorithm portrayed here is particularly skilled for concealing the secret image. 
The outcomes are organized in Table. 3 and Table.4.

\section{CONCLUSION}

This work started with the objective of analyzing the performance of processing of images in hexagonal grid. Since there is no hardware to acquire the hexagonal images, for processing the images in hexagonal grid the conversion has to be done. In this work the conversion is performed using alternate pixel suppressal method.

Table 3 MSE and SSIM between secret image before hiding and the secret image extracted.

\begin{tabular}{|c|c|c|}
\hline \multicolumn{3}{|c|}{ Cover Image : waterlilly.jpg } \\
\hline Secret Image & MSE & SSIM \\
\hline Flower.jpg & 0 & 1 \\
\hline Lena.jpg & 0 & 1 \\
\hline Barbara.jpg & 0 & 1 \\
\hline Mandrill.jpg & 0 & 1 \\
\hline Fruits.jpg & 0 & 1 \\
\hline
\end{tabular}

Table 4 MSE and PSNR between cover image and the stego image for different secret images.

\begin{tabular}{|c|c|c|}
\hline \multicolumn{3}{|c|}{ Cover Image : waterlilly.jpg } \\
\hline Secret Image & MSE & PSNR \\
\hline Flower.jpg & 0.05 & 61.54 \\
\hline Lena.jpg & 0.04 & 62.52 \\
\hline Barbara.jpg & 0.04 & 62.52 \\
\hline Mandrill.jpg & 0.04 & 62.52 \\
\hline Fruits.jpg & 0.04 & 62.52 \\
\hline
\end{tabular}

For analyzing the performance of hexagonal image processing, we have considered two important areas of image processing, the image compression and image denoising. Wavelet Transform based algorithms in hexagonal grid images is examined. The performance is excellent for hexagonal grid images. The performance is looked at utilizing MSE and PSNR.

In image denoising, wavelet thresholding based method in hexagonal grid is proposed. A new method for thresholding, named as "JeevKrish" is introduced. This thresholding method is applied to images in hexagonal grid. For the performance analysis we have used MSE and PSNR. This method is found to be better than the processing images in conventional square grid.

We have additionally proposed aalgorithm named as 'JK algorithm' for mimicking pseudo hexagonal structure and a technique for imagehiding utilizing the pseudo hexagonal structure image, which is produced utilizing the JK algorithm. The created pseudo hexagonal structure image fulfills two significant properties of hexagonal design. That is, every pixel has precisely six encompassing neighbors and the equidistance property of hexagonal pixels

The image concealing utilizing pseudo hexagonal picture gives better outcomes. Since we conceal the whole bits of secretimage utilizing the proposed method, it is fit for saving the MSE, PSNR and SSIM index of the secret image.

The above mentioned works are simulated using MATLAB. From the results obtained for all the proposed method, it is obvious that the hexagonal representation outperform the conventional image representation. So, we opine that this work will be an inspiration to other researchers in this field for further exploring the possibility of image processing in hexagonal grid.

Hexagonal representation of images helps us to make the computer vision close to human vision. An image acquiring hardware with hexagonal grid sensors are required for acquiring the hexagonal images. As there is no hardware to acquire hexagonal images, the future research may be in the field of developing sensors with hexagonal grid. This may lead to the new world with computer vision as close to human vision. So, hexagonal image processing can be considered as a missing stone to computer vision.

\section{REFERENCES}

1. H. S. M. Coxeter, "Introduction to geometry", John Wiley \& Sons. Inc. New York, 1969

2. Xiangjian He and WenjingJia, "Hexagonal Structure for Intelligent Vision", IEEE International Conference on Information and Communication Technologies, pp. 52-64, 2005

3. K. PramodSankar, T. Sanjay and E. G. Rajan, "Hexagonal Pixel Grid Modeling and Processing of Digital Images Using CLAP Algorithms", International Conference on Systemics, Cybernetics and Informatics, pp.327-336, 2004

4. Jeevan K. M. \&Krishnakumar S. "Compression of images represented in hexagonal lattice using wavelet and Gabor filter". Proceedings of 2014 IEEE International Conference on Contemporary Computing and Informatics, pp 609-613, 2014.

5. Jeevan K. M. \&Krishnakumar S. "An algorithm for wavelet thresholding based image denoising by representing images in hexagonal lattice", Journal of applied research and technology, vol.16, issue. 2, pp. 103-114, 2018.

6. C. A. Wuthrich and P. Stucki, "An algorithmic comparison between square- and hexagonal-based grids". CVGIP: Graphical Models and Image Processing, vol.53, issue. 4, pp. 324-339, 1991.

7. Yabushita A., Ogawa K. "Image reconstruction with a hexagonal grid". Proceedings of the IEEE Nuclear Science Symposium Conference Record, Norfolk, VA, USA, Vol. 3, pp. 1500-1503, 2002.

8. Jeevan K. M., Krishnakumar S. "An Algorithm for the Simulation of Pseudo Hexagonal Image Structure Using MATLAB". International Journal of Image, Graphics and Signal Processing, vol. 6, pp.57-63, 2016.

9. Jeevan K. M., Krishnakumar S. "Image hiding technique using a pseudo hexagonal structure approach", International Journal of Computers and Applications (T\&F), https://doi.org/10.1080/1206212X.2018.1438037, 2018 .

\section{AUTHORS PROFILE}

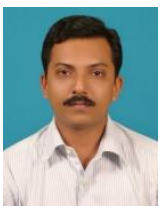

Jeevan K. M. was born in Kerala, India on 28th may 1977. He obtained his Bachelor of Technology in Electronics and Communication Engineering from Rajiv Gandhi Institute of Technology, Kottayam, Kerala, India in 1999 and Master of Technology in Electronics and Communication from College of Engineering, Trivandrum, Kerala, India in 2002.He was awarded with Ph.D. in Image Processing in 2019. He worked as a research fellow in Centre for Development of Advanced Computing (C-DAC), Trivandrum, Kerala, India and as a Hardware Design Engineer in many Multinational Companies. Currently he is associated with the department of Electronics and Communication in GITAM Deemed to be university, Bangalore, India. . He has published papers in international journals and presented papers in national and international conferences. His areas of interest include signal processing and image processing. 


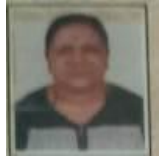

Padmaja V. K. completed her B.Teech in Electronics and Communication Engineering from Osmania University, Hydrabad, India and M.Tech in VLSI design and Embedded system from VisveswaraihTechnological University, Karnataka, India. She completed her P.hD. in Image Processing in 2019. She has more than 22 years of teaching and research experience. Her areas of interest include signal processing and image processing and VLSI

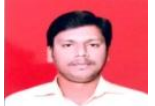

Anne Gowda A. B. completed his B.Tech inTelecommunication Engineering in 2011 and M.Tech in Signal Processing in 2014.He has more than 6 years of experience in teaching. Now he is pursuing his P.hD. in Bio-Medical image processing. His area of interest includes Digital Signal Processing and Digital Image Processing.

S. Krishnakumar was born in Kerala, India on 28th May, 1964. He completed his M.Sc. in Physics with Electronics specialization in 1987 and was awarded with Ph.D. in Thin Film Devices in 1995 from Mahatma Gandhi University, Kottayam, Kerala, India. HereceivedM.Tech. in Computer Science from Allahabad Agricultural Institute - Deemed University (renamed as Sam Higginbottom Institute of Agriculture, Technology and Sciences) in 2006 and also completed MCA from IGNOU in 2010.

He has 23 years teaching experience in Electronics and Computer Science subjects for graduate and post-graduate courses. Currently he is the Regional Director of University College of Applied Sciences, Edappally, Kochi under Mahatma Gandhi University, Kottayam. He has 11 publications in International Journal and Conferences. His areas of research include Thin Film Electronic Devices, VLSI Design and Image Processing. Dr. Krishnakumar is an Associate Member of Institution of Engineers, India. He was a member of Board of studies of University of Calicut and a member of Academic Council of Mahatma Gandhi University, Kottayam for 4 years. 